ALTERIDADES, 2021, 31 (61): Págs. 81-91

https: / / doi.org/ $10.24275 /$ uam/izt/dcsh/alt/2021v31n61/Calvo

\title{
Moviendo el orgullo: movimiento social de los jóvenes nasa y misak en Cali, Colombia*
}

\author{
Moving pride: \\ The social movement of Nasa and Misak youth \\ in Cali, Colombia
}

VALERIA ALEJANDRA CALVO ORTIZ**

BEATRIZ EUGENIA RIVERA PEDROZA***

\begin{abstract}
This article aims to show the characterization of the struggles and the ways in which emotions are revealed in the movements of indigenous youth in the city of Cali-Colombia, specifically in the Nasa and Misak communities. This research, which uses qualitative tools, involved the application of in-depth interviews and participant observation of young members of the indigenous movement in the city, all of them migrants. Some of the results show that emotions, which can be considered political, such as shame, indignation, and pride, are devices that support or impede mobilization. These emotions strengthen their various forms of protest, consolidate their epistemological struggles, and preserve the essence of their identity through collectively achieved political processes.
\end{abstract}

Key words: Mobilization, shame, indignation, protest, motivation, emotions

\begin{abstract}
Resumen
Este artículo pretende caracterizar las luchas y las formas en las que se develan las emociones en los movimientos de jóvenes indígenas de la ciudad de Cali, Colombia, específicamente de las comunidades nasa y misak. La investigación, de corte cualitativo, involucró la aplicación de entrevistas en profundidad y observación participante con integrantes jóvenes del movimiento indígena en la ciudad, todos ellos migrantes. Algunos resultados indican que las emociones, que pueden ser consideradas políticas -como la vergüenza, la indignación y el orgullo-, son dispositivos que sostienen o impiden la movilización. Estas emociones fortalecen sus diversas modalidades de protesta, consolidan sus luchas epistemológicas y preservan la esencia de su identidad a través de procesos políticos alcanzados de manera colectiva.
\end{abstract}

Palabras clave: movilización, vergüenza, indignación, protesta, motivación, emociones

\footnotetext{
* Artículo recibido el 16/01/20 y aceptado el 04/06/20.

** Universidad Cooperativa de Colombia, Cali. Carrera. 73 \#2a-80, Cali, Valle del Cauca <beatriz.riverap@campusucc.edu. CO>. ORCID: 0000-0001-5795-7776

*** Universidad Cooperativa de Colombia, Cali <valeriacalvo17@gmail.com>.
} 


\section{Introducción}

$\mathrm{E}$ 1 artículo tiene como propósito mostrar los resultados obtenidos en la investigación: "Caracterización de los nuevos movimientos sociales de jóvenes en las comunas 17,18 y 22 de la ciudad de Cali" de la Universidad Cooperativa de Colombia, campus Cali. Tuvo por objetivo identificar la lucha y la forma como aparecen las emociones en uno de los movimientos encontrados: el de los indígenas jóvenes ubicados en la ciudad, en concreto los nasa y misak, aunque debe aclararse que en ese territorio también confluyen otros grupos como los yanaconas, pastos, nukak. Según el Departamento Administrativo Nacional de Estadísticas (DANE) (2011) del 100 por ciento (9466 personas) de la población indígena que habita en la ciudad la comunidad nasa es mayoritaria y participa con más de la cuarta parte de la población (27.4 por ciento), y los misak se ubican en cuarto lugar con el 15.1 por ciento.

Según Motta (2010), la causa principal de la inmigración de la población indígena de los cabildos indígenas en Cali es la búsqueda de trabajo que no hay en los territorios de origen. También hay que resaltar que en la historia más reciente del país la violencia y el desplazamiento forzado es una forma adicional de movilidad especial para todos los cabildos. Otra causa de migración que respondió a los intereses de la investigación se relaciona con las oportunidades educativas brindadas por la ciudad, que cuenta con los centros universitarios de más alta calidad en la región.

Cali se ha establecido como la urbe más importante del suroccidente del país y se distingue por su diversidad étnica. A ella llegan los inmigrantes buscando nuevas oportunidades, es un lugar donde confluyen las luchas y donde se visibilizan las resistencias del suroccidente colombiano.

De acuerdo con Archila "los movimientos sociales son una forma de acción colectiva que reaccionan a una serie de situaciones como lo son: injusticias, desigualdades o exclusiones" (Archila, 2003: 74). En esta perspectiva se inserta la motivación del movimiento social indígena, el cual pronuncia sus resistencias en lo urbano, desde lo urbano y, en ocasiones, en contra de los valores urbanos. El movimiento social permite entender la identidad del joven indígena y sus luchas cotidianas en diferentes contextos.

Las investigaciones revisadas sobre los movimientos sociales reconocen esfuerzos de hombres y mujeres que han ejercido acciones colectivas procurando las transformaciones profundas de las injusticias sociales. Aunque se han realizado innumerables investigaciones $y$ se ha teorizado sobre el tema, es preciso reconocer que aún faltan por identificar dinámicas muy locales y propias de los movimientos que no logran ser explicados desde propuestas funcionales $\mathrm{y}$ estructurales (como la de Smelser [1962], para quien los movimientos demuestran un desequilibrio de la estructura, los recursos o sistema de medios que impulsa la acción colectiva).

Teóricos como Santos (2006), Quijano (2004), Escobar y Osterweil (2009), que se acercan a las particularidades de los movimientos latinoamericanos, y en especial a los movimientos indígenas, obligan a deconstruir una sola forma de comprender estas acciones colectivas en contextos diferentes a los europeos y a los norteamericanos. En América Latina, los campesinos, las comunidades étnicas (afros e indígenas), los ambientalistas y las feministas son actores que gestionan los cambios con perspectivas subjetivas arraigadas a los territorios y de manera interseccional. Es Melucci (1999), en el texto Acción colectiva, vida cotidiana y democracia, quien nos introduce a la comprensión de la subjetividad en el campo de la lucha social, y lo que nos ha permitido aproximarnos a los movimientos latinoamericanos es, como lo plantea Santos (2001), tal vez reconocer algunas de las características de los considerados nuevos movimientos sociales, sin necesariamente pretender acotar en ese concepto las particularidades que tienen los territorios y sus formas de reconocer sus democracias.

Recurrir a la subjetividad permite también el ingreso a las emociones, y quizá sean ellas y sus peculiaridades en el contexto las que complementen eso que se escapa a un concepto universal y excluyente de los movimientos sociales. Para Marín-Posada y QuinteroMejía (2017) las emociones políticas se aprenden en la experiencia con los otros, poseen varios rasgos que les otorgan un carácter público y no meramente individual, porque se construyen en la vida social, en la esfera pública; están soportadas en creencias y juicios y tienen un contenido evaluativo y, además, se aprenden y se cultivan. Por lo tanto, están en el sustrato moral de todos los sujetos y afectan la vida con los otros, es decir, la vida política. Las emociones políticas se aprenden del lado de las creencias y referentes morales que se apropian y se sienten en la cultura.

Para Elster (2002: 73) "algunas emociones son esencialmente sociales. Vienen provocadas exclusivamente por creencias que hacen referencia a otras personas. Nadie siente envidia de las aves porque pueden volar, 'la envidia sólo se produce entre un hombre y otro"'; Camps (201 1: 30) señala que las emociones son disposiciones mentales que generan actitudes. Son modos de reaccionar ante la realidad que, a su vez, provocan otros tantos modos de actuar en ella. 
Según Jasper (2010), la propuesta de investigación que integra conceptos sobre las acciones colectivas desde propuestas estructurales y culturales se ha ubicado en extremos que, en ocasiones, olvidan las dinámicas intersubjetivas que son las que nos permiten, acaso, comprender en profundidad los objetivos de los movimientos sociales. Estos procesos podrían estar alimentados por las emociones, las cuales pueden convertirse en el aliento que obstaculiza o favorece la movilización y, posteriormente, el cambio deseado. Según Poma, Baudone y Gravante (2015: 27) las emociones en los procesos que se dan en el interior de los movimientos sociales permiten comprender cómo y por qué las personas se involucran en una acción colectiva y hasta dónde están dispuestos a llegar. En el campo de las emociones y los movimientos sociales se encuentran referentes importantes como Jasper y Poulsen (1995) y Flam y King (2005), quienes han sido pioneros en el desarrollo de una propuesta teórica útil para pensar el papel protagónico de las emociones en las acciones colectivas y, sobre todo, en aquellas que tienen una implicación política.

Definir el contexto urbano de los jóvenes indígenas es importante para entender sus luchas sociales. Para Del Popolo, Ribotta y Oyarce (2009) los indígenas urbanos son aquellos que residen en espacios urbanos. En las zonas urbanas se registra mayor presencia de los servicios del Estadoy, por lo tanto, mayor cobertura de centros de salud y mayor acceso a la educación formal, por lo cual se presume que los índices de mortalidad son más bajos. Sin embargo, Del Popolo, Ribotta y Oyarce (2009) señalan que las brechas de desigualdad para los indígenas son recurrentes, por lo que aun residiendo en espacios urbanos hay sobremortalidad y poco acceso a la educación. Los autores dicen que la discriminación social de los indígenas que viven en la ciudad se fortalece mucho más cuando los migrantes indígenas se instalan en zonas precarias donde no hay centros de salud o no son suficientes y donde prevalecen altos índices de marginalidad. Los jóvenes indígenas que arriban a la ciudad llegan sin ningún recurso, en sus territorios de origen, la misma tierra les proporcionaba los alimentos, pero, en las urbes, se encuentran con luchas alrededor de los derechos humanos, donde la universidad es una puerta para reivindicar lo ancestral y valorar lo propio. Además, para comprender a esta población, es necesario defi- nir el concepto de joven y para ello recurrimos a Reguillo (2012), quien afirma que:

Los jóvenes [...] son vistos como sujetos con competencias para referirse a las entidades del mundo, es decir, como sujetos de discurso y como agentes sociales, con capacidad para apropiarse de (y movilizar) los objetos tanto sociales y simbólicos como materiales. En otras palabras, se les reconoce el papel activo en su capacidad de negociación con las instituciones y estructuras [p. 30].

Los jóvenes indígenas que realizan la transición de lo rural a lo urbano asumen el reto de agenciar y movilizar los recursos que como comunidad étnica necesitan, Reguillo precisa las competencias sociales que emergen del joven, de donde se deriva la importancia del papel de éste en las luchas de la movilización social, pues a través de los jóvenes hay una esperanza de transformación. Los jóvenes sobre los que versa esta investigación han sido parte de procesos que buscan reivindicar sus dinámicas comunitarias a partir de su profesión o su rol en los barrios a los que migraron en la ciudad. Los migrantes del Cauca manifiestan experimentar discriminación en la universidad y a veces en los barrios, sin embargo, salen de su pueblo de origen -algunos en busca del conocimiento para llevar la teoría a las vivencias de su propio territorio-, y en su discurso siempre se evidencian los propósitos de volver a sus municipios, en lo posible fortalecidos y de esta manera orientar a otras personas.

La investigación fue cualitativa descriptiva, se hicieron entrevistas a profundidad y observación participante con jóvenes del cabildo Alto Buena Vista y la universidad pública de la ciudad y en la sede de la misma universidad, que se ubica en otro municipio (Santander de Quilichao en el Departamento del Cauca). Se seleccionaron jóvenes que pertenecían a comunidades indígenas, fueran activos en la propuesta del movimiento y mostraran disponibilidad. Las personas que aceptaron participar motivados por el tema de la investigación fueron hombres de entre 20 y 29 años. El proceso empezó indagando las formas de protesta, los objetivos de la movilización, el contexto y la manera en que se organizan; estas categorías se analizaron y con base en los datos que afloraron se volvió a problematizar y a reflexionar los hallazgos (identidad política y emociones). ${ }^{1}$

1 En la primera etapa se realizó una codificación abierta donde se analizaron las entrevistas con el fin de descubrir minuciosamente las ideas, sentidos y percepciones contenidos en el discurso. Para ello, se extrajeron códigos in vivo (frases o palabras exactas de los participantes), los cuales, ya establecidos con el método comparativo, permitieron contrastar una y otra vez los códigos previos, con los nuevos códigos obtenidos. A partir de éstos se elaboraron conceptos de ocurrencias específicas de los datos, suministrados en los memos del programa donde se describen los vínculos, las secuencias y los pensamientos de las categorías que van surgiendo en el ejercicio. Mediante la codificación axial se empezaron a vincular y 


\section{Hallazgos}

El siguiente apartado, como ya se mencionó, es el resultado del doble análisis y en él se plasman las tres categorías iniciales y las categorías obtenidas del segundo momento metodológico.

\section{La movilización (movimiento de ideas)}

Desde su identidad, los nasa y los misak que habitan en la ciudad se comunican con la naturaleza para tener una relación de equilibrio y armonía con su familia y su entorno. Consideran la lengua materna como el origen y la final de su identidad y cosmovisión indígena, por lo que estudiarla, conservarla y transmitirla es una tarea obligatoria de todos los días. El cabildo urbano ofrece clases de lectura y escritura todos los viernes después de la asamblea general, lo cual abre la posibilidad de asistir a los cursos ofertados por el cabildo de la universidad pública (Martínez, 2015)

En la movilización es donde se gestan las ideas, los objetivos y las formas de visibilizar las concepciones individuales y colectivas. Cada movimiento social tiene una historiay, dependiendo del contexto y la cultura, agencia diferentes formas de reflexión y organización; así lo señalan Loaiza, Patiño y Alvarado:

La resistencia indígena configura un proceso de autorreconocimiento de su historia, cultura, contexto, políticas de vida e intereses, que dan sentido propio a las situaciones vividas y a los horizontes de posibilidad que se pueden construir. Caracterizada por la generación de espacios y procesos de encuentro, propiciando la problematización, el diálogo, los acuerdos, la sensibilización, la capacitación, la gestión y protección de la vida-territorio-cultura [2011: 31].

En la movilización, los jóvenes indígenas realizan un proceso de reconocimiento y valor hacia su comunidad de origen, pues en la ciudad asumen el reto de vivir una cultura de la soledad, enfrentando situaciones económicas extremas (como largas jornadas de ayuno) que les llevan a movilizarse y así mejorar sus condiciones de vida (tener acceso a comida).
Por otro lado, el lugar donde se gestan las ideas es el Cabildo Indígena Universitario (CIU), donde se discuten las problemáticas actuales y todos participan de manera activa construyendo reflexiones y posibles alternativas. El Cabildo es un espacio que, aunque se inserta dentro de la universidad, es el vínculo con la organización indígena de origen, donde se sienten acompañados, y el cual les permite tejer la palabra e ideas de resistencia. Así lo afirma el joven nasa:

Para un indígena enfrentarse a una economía, a una cultura diferente es muy difícil, pero la necesidad te lleva a eso... La gente se deja llevar mucho por los medios de comunicación, pero no se dan cuenta de las realidades y las luchas de los pueblos indígenas. Todos los jóvenes tienen la capacidad de ir a la universidad, pero hay muy poca posibilidad, la universidad no está hecha para todas las personas, la universidad te sectoriza. Preocupa mucho el tema económico cuando uno entra en la universidad

[...].

Llegar al civ es conocer pensamientos de otros pueblos indígenas, de otras personas que me ayudaron a fortalecer, el tema de respeto, el tema de la integralidad, muchos no teníamos que comer, muchos sólo tomamos un café y cada uno, a veces de lo que tenían ponían para hacer una comida para todos, la vida tiene que ser comunitari[a] y desde ahí la integralidad. El llegar a la universidad me hizo valorar mi comunidad, a mí mismo, por esas vivencias [J. N., 6 de diciembre de 2017].

Por lo anterior, la función del ciu es fundamental para que los jóvenes que llegan a la ciudad sigan movilizándose, tal como lo hacían en sus territorios. Las formas de movilización se trasladan a la ciudad, promoviendo la visibilización de los pueblos originarios y las formas de lucha ancestrales por sus derechos humanos.

Según los jóvenes indígenas entrevistados, las luchas también son epistemológicas, esto consiste en poner su saber académico en función del saber ancestral, y de este modo poder reivindicar lo propio: "Mi sueño ha sido el de ser investigador y producir textos académicos para reivindicar el conocimiento ancestral en la misma fuerza del conocimiento occidental; centrar una situación de igualdad" (J. M., 6 de diciembre de 2017). Por otro lado, puntualizan su deseo de lograr

relacionar las categorías iniciales con las emergentes, logrando evidenciar que la emoción, las motivaciones y la identidad política son elementos influyentes en la movilización. Es esencial mencionar que para lograr esta vinculación también se hizo uso de herramientas didácticas tales como el mapa conceptual, y así tener una mayor comprensión. Al terminar la primera revisión, se hizo un nuevo análisis, mediante un rastreo por los códigos y memos arrojados por el Atlas ti, con el fin de asegurar que no hubiera más categorías emergentes y que por ende se había alcanzado la saturación teórica (cuando los datos empiezan a tornarse repetitivos y no se encuentra nada nuevo). 
relaciones de igualdad: "Una de las características del porqué estudié, desde mi rama, que es la literatura, es segmentar una teoría académica, desde las artes de las escrituras. Eso sería un trabajo que aportaría desde lo académico, pero también desde lo originario" (J. N., 6 de diciembre 2017).

La ciudad es la oportunidad de visibilización de lo propio. Para ellos la medicina tradicional es igual o más importante que los hallazgos científicos de Occidente, y por ello debe ser rescatada y puesta al servicio de los más vulnerables. El joven misak indica que para él las luchas en la ciudad son lides relacionadas con la raíz del conocimiento y su legitimidad, con la resistencia que logra oponerse al sistema y rescatar lo ancestral.

En la movilización, para el joven indígena, migrar a la ciudad significa asumir nuevos retos que lo conduzcan a responderse cuestiones como ¿qué carrera estudiaré?, ¿volveré a mi territorio?, ¿conseguiré un trabajo en la ciudad? Cada interrogante se convierte en la lucha constante entre la identidad y la influencia de lo urbano. Los jóvenes indígenas que llegan a la universidad para estudiar una carrera profesional son invitados a participar en el cIU. Ante esta posibilidad, algunos deciden no participar y otros de manera activa y permanente asisten al cabildo. Para Loaiza, Patiño y Alvarado (201 1: 23), los jóvenes del movimiento juvenil indígena comparten, además de su cultura, territorio, motivaciones políticas y motivaciones racionales atravesados por la esfera de las emociones.

Loaiza, Patiño y Alvarado también mencionan que las motivaciones de los jóvenes indígenas son políticas y racionales; es ahí donde radica la necesidad y el impulso a la acción. Para ellos, las motivaciones políticas tienen que ver con el reconocimiento de la vulneración constante de derechos humanos hacia su etnia; el interés en los procesos de formación que el movimiento ofrece en temas de liderazgo, derechos humanos, participación y derecho ancestral; la necesidad de trabajar con la comunidad para generar un bienestar colectivo; la búsqueda del fortalecimiento de su identidad cultural como pueblo indígena; la conciencia del ser colectivos históricos y la necesidad de crear espacios de reconocimiento intergeneracional en los que emerja la diferencia de los jóvenes y que éstos puedan ser aprovechados para disminuir el desempleo, el analfabetismo, el consumo de alcohol, el reclutamiento forzado y la migración juvenil (Loaiza, Patiño y Alvarado, 2011).
Las motivaciones racionales se caracterizan por ser observables y objetivas. En los discursos de las entrevistas se evidenció cómo ambas motivaciones son atravesadas por las emociones de la vergüenza política y la nostalgia ancestral que empuja al joven indígena a movilizarse por sus motivaciones políticas. Un ejemplo de esta movilización es la tulpa, ${ }^{2}$ un espacio donde convergen el arte (baile), las ideas y las tradiciones ancestrales, y que se encuentra dentro de la universidad, como una forma de recuperar espacios propios y de abrir el debate.

Lo interesante es que los jóvenes entrevistados tienen una característica en común: que sus esfuerzos son concentrados en su pueblo, en su identidad y no en intereses individuales. Sus motivaciones son políticas, como se mencionaba antes, desean la libertad y la igualdad. Son jóvenes que se levantan con el saber de lo propio y no huyen del saber occidental, sino que convierten a éste en aliado para rescatar sus tradiciones ancestrales en la ciudad.

La movilización del joven indígena está en las raíces, como ellos mismos lo expresan, y las desarrollan viviendo procesos de construcción de identidad, reconociendo las tradiciones como propias y supliendo las necesidades afectivas y comunicativas con su entorno. Uno de los jóvenes misak cuenta cómo fue su proceso de desarrollo dentro de su comunidad:

Desde antes de dar a luz a ese nuevo misak empieza una serie de rituales, donde se pretende recibir en un ambiente propicio al nuevo misak. Asimismo, mientras está embarazada la mamá, ella asiste a asambleas y a diferentes actividades culturales, políticas y él bebé está ahí haciendo presencia; no ha nacido todavía, pero también está movilizándose. En las mingas del pueblo nasa, niños de seis o siete ya hacen parte de la guardia indígena y saben cómo hacer formación, tienen bastón de mandoy saben de las formas de ejercer orden dentro de una comunidad. Entonces desde muy niño uno empieza a [ser] partícipe de estos escenarios [J. M., 1 de diciembre de 2017].

En las entrevistas se encontró que la vinculación de los jóvenes indígenas con el movimiento despliega unas motivaciones tanto de la vida privada (relacionada con su desarrollo gestacional y posterior crecimiento, donde es permeado por su cultura) como de la vida pública, donde buscan participar, influir, movilizarse por los derechos colectivos y por sus motivaciones políticas. Todo esto es atravesado por la emoción.

2 Tulpa: espacio de reunión o espacio de minga. 


\section{Formas de protesta}

La protesta se distingue por ser acciones concretas, donde se visibiliza la movilización de los jóvenes indígenas, esto se da gracias a que, una vez situados en lo urbano, ellos crean estrategias para la reivindicación de sus tradiciones ancestrales, tales como la tulpa, la huerta, la minga, los cabildos, entre otras actividades que logran seducir a quienes los observan.

Las formas de protesta de los jóvenes nasa requieren acciones que a veces interrumpen la normalidad. Así lo señala uno de los jóvenes del pueblo nasa: "nosotros somos más de acción, la mano de presión que ponga entre la espada y la pared" (J. N., 6 de diciembre de 2017), por ejemplo realizar un bloqueo a la entrada de la universidad. Por otro lado, los misak, por su cosmogonía, son más simbólicos, como lo afirmaron en sus narraciones: "defender el territorio a través del diálogo, la negociación” (J. M., 1o de diciembre de 2017), un ejemplo de ello es negociar con las directivas de la universidad para llegar a acuerdos que beneficien a la comunidad indígena, ellos usan cartas, correos electrónicos o reuniones presenciales para dialogar y evidenciar las necesidades de la comunidad. Estas dos cosmovisiones se unen con el fin de especificar las formas de protestas del ciU. Uno de los misak indica que sus acciones y su forma de organización van encaminadas a lograr la autonomía. Los nasa, aunque reconocen las acciones desde el diálogo, suelen ejercer más la presión y por ello son reconocidos como fuertes en sus luchas. Así lo argumenta uno de los jóvenes:

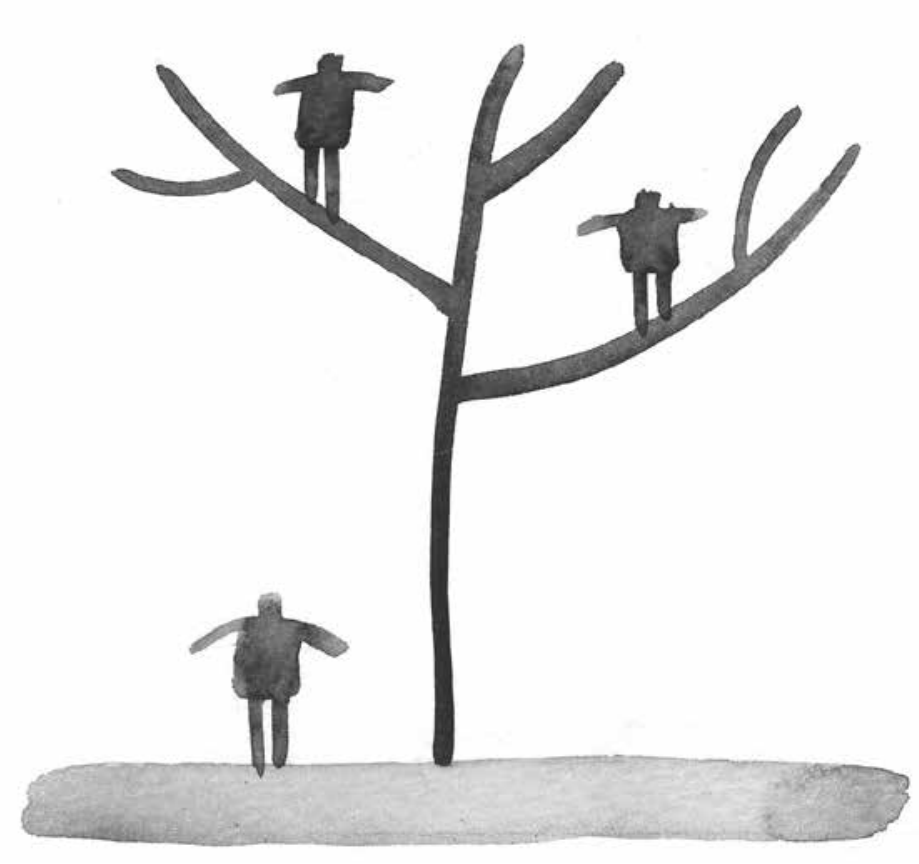

Uno ha entendido y ha vivido cómo las diferentes visiones sientan esa relación de gobierno a gobierno, pero también las relaciones de comunidad, la esencia de la espiral, la comunidad como tal, porque a veces la institucionalidad rompe con la visión de los pueblos. Entonces eso es lo que decía mi compañero: "a veces nos tratan de gobiernistas”, pero es preciso, porque es la mirada de ejercicio de ellos. Sin embargo, en otros pueblos no es bien visto ese ejercicio, aunque eso rompe con algunas esencias del pueblo. El CIU ha facilitado ese ejercicio de interactuar esas tres cosmovisiones, no sólo en el ejercicio de presión, sino también de negociación y diálogo para interactuar y compaginar las relaciones de conocimientos... [J. N., 6 de diciembre de 2018].

Uno de los entrevistados advierte que las tradiciones de sus territorios de origen son traídas a la ciudad con el fin de resistir a las dinámicas occidentales que ofrece la academia, además de preservar su identidad:

Una de las formas de movilización ha sido recuperando espacios dentro de la universidad, pero también ir recuperando prácticas culturales dentro de estos espacios. Por ejemplo, en la ciudad de Cali nunca se ha visto una tulpa como se hace en la Univalle y todo para recordarnos nuestra identidad como indígenas y que allá tenemos que volver, pero también hay otros espacios que generan que los jóvenes no pierdan el tema del aprecio a la tierra a cultivar por eso en el 2012 se creó una huerta universitaria dentro de la universidad para no olvidarnos que nosotros somos de la tierra [J. N., 1ㅇ de diciembre de 2020].

\section{Lo que emerge...}

Del discurso resultado de las entrevistas, de las observaciones participantes y de los documentos sistematizados se destacan las siguientes categorías:

\section{La identidad política del joven indígena en la ciudad}

Giménez (2000: 28) afirma que la identidad se ha definido como el conjunto de repertorios culturales interiorizados a través de los cuales los actores sociales demarcan sus fronteras y se distinguen de los demás, dentro de un espacio históricamente específico y socialmente estructurado. Los pueblos originarios han adquirido conciencia de que los intercambios sociales consolidan su propia identidad, donde se busca practicar la sabiduría ancestral y se vive colectivamente. A través de esos espacios, la identidad del joven indígena 
se construye y se reconstruye. Por lo tanto, dejar el territorio y vivir en la ciudad no es tarea fácil, pues irrumpe su relación con la cotidianidad, chocando con las lógicas occidentales que deben enfrentar solos.

Muchos de los jóvenes salen de sus territorios con el fin de estudiar en la universidad, pensando en el futuro o, como lo dicta la tradición, centrando la atención en lo que debe ser rescatado y reivindicado del pasado. Para una mejor comprensión de este panorama, hay que entender que la identidad política de los jóvenes se remonta a la década de 1980, con la preocupación del sacerdote y líder comunitario Álvaro Ulcué Chocué, quien consolida el movimiento juvenil indígena. Su enseñanza fue tan sólida que su legado permanece en los jóvenes indígenas. Loaiza, Patiño y Alvarado lo ponen de manifiesto:

El padre Ulcué empieza a preocuparse por las condiciones de vulnerabilidad, pobreza, discriminación y violación de derechos humanos que generaba la guerra para el pueblo nasa y especialmente para los y las jóvenes; en dicho contexto el sacerdote promovió la organización de grupos juveniles de 17 cabildos ubicados en el Norte del Departamento del Cauca.... A partir del asesinato del padre Ulcué, en 1989, el movimiento inicia un proceso de resignificación para los jóvenes como sujetos con capacidad para la transformación de sus familias, comunidades y regiones [2011: 19].

En relación con las luchas que asumió el padre Ulcué, en la actualidad muchos de los jóvenes indígenas asumen un rol activo dentro y fuera de su territorio, lo sostiene el entrevistado quien terminó sus estudios de pregrado en la universidad pública y posteriormente regresó a su territorio de origen: "vemos que la mayoría de las personas que protestan y luchan más fuerte son los jóvenes" (J. N., 1o de diciembre de 2017). Afirma también que los jóvenes, en comparación con el resto de la comunidad, son quienes tienen pensamientos más similares a los mayores de 50 años, que en su juventud pensaron en el futuro y lucharon por el buen vivir de las futuras generaciones "Los mayores de 50 hacia arriba nos han dicho que los jóvenes de la actualidad ojalá no vayan a perder el pensamiento, porque son casi los mismos pensamientos que ellos tuvieron cuando también comenzaron a hacer la recuperación de tierras" (J. N., 1 de diciembre de 2020).

Los jóvenes indígenas que llegan a la ciudad se unen en una identidad política a través del cIU, donde convergen jóvenes de diferentes pueblos, logrando ejercer acciones colectivas para dar respuesta a problemáticas en el cabildo: universitarias, institucionales y de comunidad. Hablamos de identidad política porque hay un solo sentir de vivir comunitariamente, de apoyo y de resistencia ante los procesos organizativos.

\section{Emociones en los jóvenes nasa y misak}

Las emociones en los jóvenes indígenas configuran el conjunto de creencias forjadas cultural y socialmente respecto a su cosmovisión. Éstas se afirman y reafirman en el vínculo, en el contacto y las relaciones en torno al gran movimiento.

En las narraciones se enfatizaron algunas emociones que pueden tener la función de sostenedoras de la dinámica de las luchas indígenas. Estas emociones son: la vergüenza, la indignación y el orgullo, las cuales afectan e influyen en la movilización.

El sentimiento de vergüenza está estrechamente vinculado a los orígenes del sentido moral; consiste en el sentimiento derivado de la caída de la imagen que uno tiene de sí mismo, la pérdida de reputación, el descrédito ante algún otro o ante la sociedad. El sentimiento de vergüenza se genera cuando existe un ojo que mira y juzga a la persona. La vergüenza se relaciona con el desagrado o el disgusto que manifiesta la sociedad hacia quienes quedan fuera de la normalidad que ella misma establece y preserva, razón por la que quienes se encuentran en tal situación se ven estigmatizados y marginados (Camps, 2011). En la vergüenza ajena, presentir al otro incluye el sentimiento de lo colectivo, y esto no tiene que ver con la cercanía, pues siempre en nuestra mirada está la comparación. Según Gil:

en esa comparación cuando algo que no considerábamos vergonzoso anteriormente, puede pasar a parecernos tal cosa. Uno puede incluso llegar a sentir lo que denominamos "vergüenza ajena" cuando opinamos que algo que ha hecho otra persona está mal. [...] Así pues, queda claro que el sentimiento de vergüenza hunde sus raíces en nuestras relaciones con los demás [2013: 12].

Por otra parte, dicho sentimiento también se encuentra estrechamente vinculado con el respeto, tanto hacia los otros como hacia uno mismo.

En cambio la indignación, según Camps (2011), estaría más asociada con la percepción de una injusticia, es el enfado por el no reconocimiento o la falta de respeto. En la narración de los jóvenes de los movimientos indígenas se resaltaron dos emociones que se vuelven recurrentes en sus descripciones: la vergüenza y la indignación. La vergüenza la sienten como consecuencia de la discriminación y la exclusión por parte de lo que consideran la cultura occidental, pero también la vergüenza de no defender con fuerza 
sus costumbres, hasta pasar por la vergüenza ajena, generada por los comportamientos de quienes, siendo representantes del saber ancestral, tienen prácticas poco éticas, contrarias a sus valores, en el escenario público nacional. En la primera forma de vergüenza es evidente el menosprecio histórico, en cambio en la indignación adquieren conciencia de haber sido violentados por años (actos inmorales), pero también buscan aproximarse al orgullo que provoca el reconocer sus riquezas. En lo que Gil (2013) considera como vergüenza ajena es posible identificar cuando se plantea que el comportamiento de otras personas no está bien, y así lo expresan:

Llegan, digámoslo un ejemplo, a gobernadores, llegan a concejales o de pronto a alcaldes o a puestos más grandes, se olvidan y ya empiezan a pensar individualmente y tenemos un ejemplo muy grande aquí en Colombia, un compañero indígena que es senador [...] los líderes y las personas que representan la comunidad están pensando en el momento y no están pensando en el futuro de la comunidad y ahí es pues que vemos esa preocupación [J. N., 1 o de diciembre de 2020].

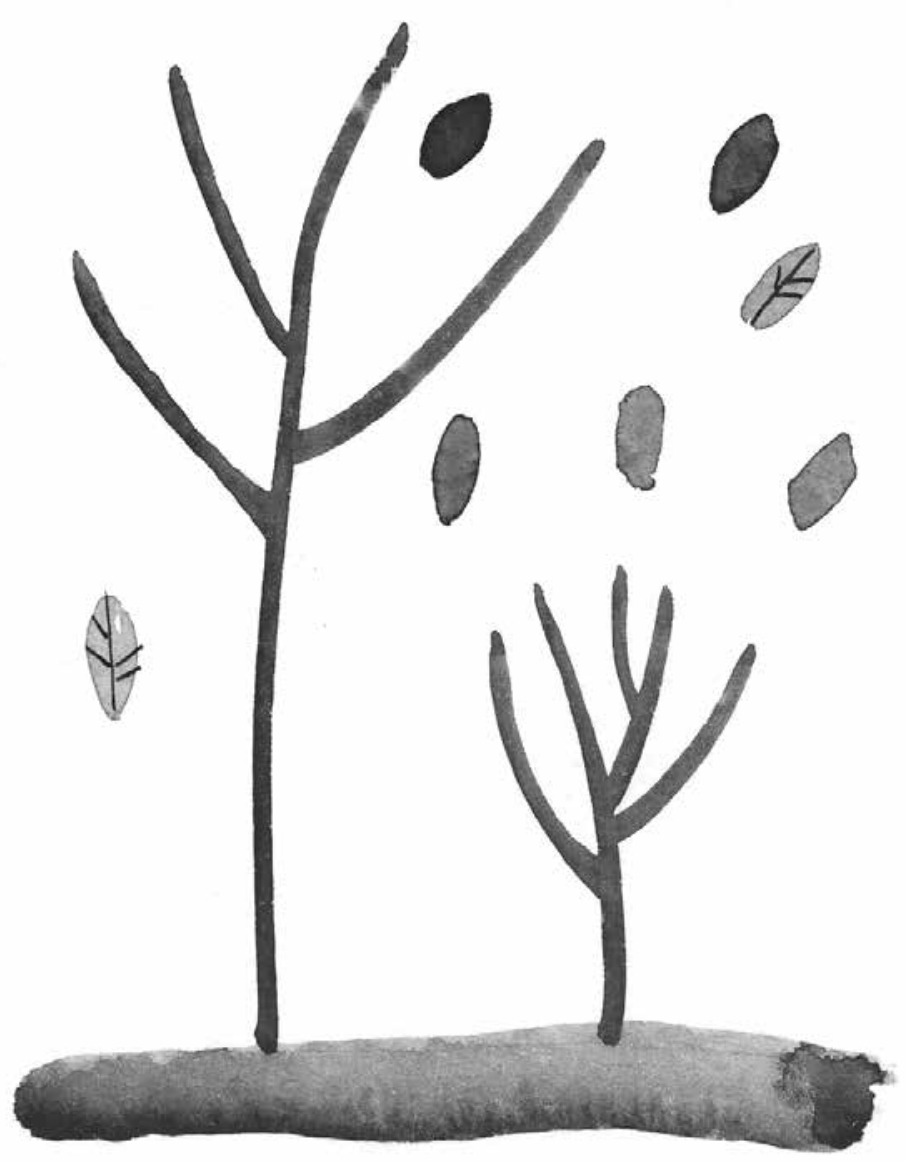

En las versiones de vergüenza e indignación se evidencia una intensa relación con el honor, identificando la relación que tiene la emoción con las construcciones colectivas: "yo creo que eso ha generado de que la sociedad actual está como está y que también piense de que las comunidades indígenas ya no son tan fuertes como hace algunos años pasados (J. N., 1ㅇ de diciembre de 2020].

Recogiendo los comentarios de Elster (2002), las emociones se producen por las creencias sobre las acciones y a su vez las motivaciones que podrían estar impulsando (como la vergüenza, la ira, la compasión y el miedo); otras aluden a las posesiones (como la indignación, la envidia y la malicia); por último están las ligadas a rasgos de carácter, como el odio y el desprecio. En términos generales, las emociones contienen creencias que permiten que ellas mismas afloren o se inhiban. Sin embargo, la vergüenza se enmarca en emociones que están ligadas a la "conciencia propia".

La vergüenza es provocada por la desaprobación, llena de desprecio o repugnancia que otros muestran por algo que uno ha hecho. Por lo general, se necesita la presencia de un otro. Sin embargo, Elster (2002) señala que la vergüenza es un potente regulador del comportamiento, puesto que se evita hacer cosas que podrían hacerte sentir avergonzado aunque esas cosas sean propias de tu identidad. Además, argumenta que la vergüenza es una experiencia intensamente dolorosa cuando no es prevista (Elster, 2002). Los jóvenes que migraron a la ciudad y que han confrontado su saber con el encontrado sienten más descontento cuando descubren el comportamiento de algunos representantes del movimiento que olvidan las resistencias y se acomodan a valores que no son los propios. Una mayor conciencia de su identidad; de alguna manera estos jóvenes saben que la vergüenza que acompaña a sus pueblos no es nada inesperado y, por el contrario, les produce indignación reconocerlo; para ellos es fundamental seguir el legado de sus ancestros, y determinan que gracias a sus luchas ellos ahora se reconocen en un territorio y poco a poco rescatan los saberes perdidos.

El orgullo describe la satisfacción personal experimentada por la consecución de un logro propio o ajeno (Valdez, Huerta y Díaz, 2014). La ancestralidad, la tierra, lo autónomo son experiencias que describen la complacencia y la alegría indígena. Luchar por lo propio se convierte en el motor para exhibir su orgullo en la ciudad, generando identificación con todos aquellos que se unen a una misma causa. El cIU, por ejemplo, es el lugar de encuentro y donde se muestra el orgullo que cada uno tiene de pertenecer a sus 
pueblos, de no saberse aislados, donde se consolidan las ideas que representan sus cosmovisiones; dicho proceso reafirma su identidad como indígenas.

Para Elster (2002: 179) el orgullo "es una emoción positiva provocada por una creencia acerca de una acción propia”. Las tradiciones propias de los pueblos indígenas generarán en estos jóvenes indígenas emociones de vergüenza o de orgullo dependiendo del nivel de indignación que el desprecio hacia sus pueblos les genere. La vergüenza debilita la identidad, pues es un sentimiento de desaprobación respecto a lo propio (ideología, vestimenta, procesos), mientras que la vergüenza ajena puede estar más cerca de la indignación, y esto puede explicarse porque el líder que debería representar sabiduría ancestral no lo hace. El orgullo, entonces, fortalece la identidad; es el punto de equilibrio entre la vergüenza y la indignación.

\section{Conclusiones}

Las narraciones muestran un interesante recorrido por los movimientos sociales, las modalidades de protesta, la identidad y, por último, las emociones (vergüenza, indignación y orgullo) y la forma en que éstas se convierten en el dispositivo esencial para sostener o agotar las luchas ancestrales de los jóvenes de comunidades indígenas que migran a la ciudad.

El cóctel entre emociones como la vergüenza, la indignación y el orgullo es el motor de la participación activa del movimiento indígena, los jóvenes que llegan a Cali lo saben y por esta razón propician el reconocimiento de estas emociones para sostener la motivación de las personas que llegan a los barrios o a la universidad, es la garantía de que las luchas de sus ancestros no se pierdan. Estas relaciones no son evidentes a simple vista, pues se habla de culturas con construcciones sociales diferentes a las occidentales y, por lo tanto, no se dejan leer desde parámetros universalizados.

Existen formas diferentes de protesta de los misak y de los nasa, y esto tal vez se deba a que las emociones que se gestionan en cada una de las comunidades son distintas. Esto es, aunque los jóvenes de ambas comunidades son conscientes de la relación que su cultura ha tenido con la de Occidente, pueden generar respuestas diversas. Las respuestas manifestadas por los jóvenes en las dos comunidades pueden estar relacionadas con el shock moral, concepto utilizado por Jasper (2010), que se origina en cada comunidad y que, al tener una mezcla diferente, produce reacciones distintas favoreciendo la incorporación y el mantenimiento de individuos en el movimiento.
Quintero y Vasco (2007), retomando a los filósofos Hudson, Hare, Tugendhat y Habermas, reconocen que en la vida cotidiana todas las justificaciones morales poseen en ellas mismas una intención comunicativa. Por lo tanto, los jóvenes empiezan a identificar que para legitimar sus formas de lucha deben al mismo tiempo identificar lo que consideran que no es moral desde las construcciones realizadas en sus comunidades. Quintero y Vasco (2007) precisan que, en el pensamiento de Habermas, la vulneración a la norma se expresa a través de los sentimientos morales. En la voz del joven activista, las acciones de algunos líderes reflejan comportamientos que no constituyen parte de las normas establecidas por la comunidad, como si estos actos fueran el resultado de un contagio de la cultura a la que se resisten.

Los movimientos buscan eliminar los sentimientos negativos, la culpa, la vergüenza y la indignación, entre otros, derivados de los procesos de socialización, para reemplazarlos con emociones adecuadas que favorezcan la movilización de sus miembros, modificando las reglas emocionales del grupo (como el orgullo), que permiten valorar y reconocer sus raíces. Las emociones pueden provocar respuestas que conduzcan a la acción o a algo completamente opuesto: el sentimiento de ser incapaz de actuar, por la parálisis que producen el miedo o el terror.

Hochschild (1979) diseña su perspectiva de la gestión de las emociones a partir de la imagen de un sujeto que es capaz de sentir, que es consciente de esa capacidad y que quiere sentir. Con sus estudios demostró que las personas crean, manejan y transforman su sentir. También probó que ese trabajo emocional puede ser estratégico, pero también inconsciente.

Como se ya mencionó, el honor es lo que se juega entre la vergüenza y la indignación. Después de que el indígena experimenta vergüenza (propia o ajena), pasa a una fase de indignación donde puede experimentar que lo propio es rechazado y el honor es anulado. Por lo tanto, duele que muchos de los jóvenes indígenas que fueron concebidos en la resistencia, crecieron bajo dinámicas de lucha, de amor a lo propio y maduraron con las enseñanzas de sus mayores no actúen en consecuencia. Es por esta razón que los jóvenes indígenas en lo urbano se unen desde el saber para reclamar sus derechos; no sólo para ellos, sino también para sus pueblos en diferentes lugares del país. Se movilizan, bloquean, cuidan la universidad y vuelven a sus comunidades a transmitir el conocimiento. Aunque la vergüenza debilita, también se fortalece en acciones que incentivan el orgullo por ser indígena, por el saber ancestral, visibilizando sus tradiciones, creyendo en una esperanza para ellos y sus futuras generaciones. 
Por último, las costumbres de las comunidades indígenas, en especial las de los nasa y los misak, protagonistas de este artículo, se traducen en una vida en comunidad, siendo parte de los procesos políticos, pero sin perder la esencia y sus principios: respetar y aceptar la diferencia para lograr la igualdad, así como pensarse colectivamente y no de manera individual, rechazando todo acto que dañe a la tierra, la naturaleza, pero también al ser humano.

\section{Fuentes}

\section{Archila, Mauricio}

2003 Idas y venidas, vueltas y revueltas: Protestas sociales en Colombia, Instituto Colombiano de Antropología e Historia/Centro de Investigación y Educación Popular, Bogotá.

CAMPS, Victoria

2011 El gobierno de las emociones, Herder, Barcelona.

DANE

2011 Cuantos somos/Como vamos. Diagnóstico sociodemográfico de Cali y 10 municipios del Pacífico nariñense, AfroamericaXXI, Cali <https: / / www.dane.gov.co/files / censo $2005 /$ etnia/sys/cuantos_somos.pdf> [15 de junio de 2018].

Del Popolo, Fabiana,

BRUNO RiBotTA

y Ana María Oyarce

2009 "Indígenas urbanos en América Latina: algunos resultados censales y su relación con los objetivos del desarrollo del milenio", en Notas de Población, año XXXV, núm. 86, pp. 99139.

ELSTER, JON

2002 Alquimias de la mente, la racionalidad y las emociones, El Roure Editorial, Barcelona.

Escobar, Arturo

y Michael Osterweil

2009 "Movimientos sociales y la política de lo virtual. Estrategias deleuzianas", en Tabula Rasa, núm. 10, pp. 123-161.

Flam, Helena

y Debra KING

2005 Emotions and Social Movements, Routledge (Advances in Sociology), Nueva York.

GiL, Martha

2013 "El sentimiento de vergüenza: una aproximación desde la Ética Nicomáquea y la Retórica de Aristóteles", en Daimon: Revista Internacional de Filosofía, núm. 58, pp. 5-18.

Giménez, Gilberto

2000 "Identidades en globalización", en Espiral, vol. VII, núm. 19, pp. 27-48 <http: / / www.redalyc. org/pdf/138/13801902.pdf> [15 de abril de 2018].

Hochschild, ARlie

1979 "Emotion work, feeling rules and social structure", en American Journal of Sociology, vol. 85, núm. 3, pp. 551-575.

JASPER, JAMES M.

2010 "Social movement theory today: Toward a theory of action?", en Sociology Compass, vol.
4, núm. 11, pp. 965-976. DOI: $10.1111 /$ j.1751 9020.2010.00329.x

JASPER, JAMES M.

Y JANE D. POULSEN

1995 "Recruiting strangers and friends: Moral shocks and social networks in animal rights and anti-nuclear protests", en Social Problems, vol. 42, núm. 4, pp. 493-512<http: / /dx. doi.org/10.1525/sp.1995.42.4.03x0129y> [ 10 de febrero de 2018].

LOAIZA, JULIÁN,

JHOANA PATIÑO

y Sara Alvarado

2011 "Movimiento juvenil indígena Álvaro Ulcué Chocué: la emergencia de un nuevo sujeto social y político en el pueblo nasa: los y las jóvenes como protagonistas de la acción colectiva", en Héctor Ospina et al., Experiencias alternativas de acción política con participación de jóvenes en Colombia, Alianza Cinde-Universidad de Manizales, Manizales, pp. 17-42<http: / / bibliotecavirtual.clacso. org.ar / Colombia / alianza-cinde-umz $20130415062106 /$ Experienciasalternativas. 17-42.pdf> [2 de marzo de 2018].

Marín-Posada, Mary Luz

Y MARIETA QUINTERO-MEJÍA

2017 "Emociones políticas y mal", en Revista Eleuthera, núm. 16, pp. 101-117. DOI: 10.17151/ eleu.2017.16.7

Martínez, Ana María

2015 "Transformaciones socioculturales y prácticas comunicativas del cabildo indígena nasa alto buena vista: el caso de la comunidad nasa en el sector alto Nápoles, Meléndez en la ciudad de Cali", tesis de pregrado, Universidad Santiago de Cali, Cali.

Melucci, Alberto

1999 "Teoría de la acción colectiva", en Alberto Melucci, Acción colectiva, vida cotidiana y democracia, El Colegio de México, México, pp. 2554.

Motta, NANCY

2010 "Tejiendo la vida en la ciudad de Cali. Estrategias de adaptación e inclusión de seis cabildos indígenas urbanos", en Historia y Espacio, vol. 6, núm. 34 <https: / / dialnet.unirioja. es $/$ servlet $/$ articulo?codigo $=4016466>[20 \mathrm{de}$ abril de 2018].

Poma, Alice,

Marco Baudone

y Tommaso Gravante

2015 "Más allá de la indignación. Una propuesta de análisis desde abajo del movimiento de los indignados”, en Espiral, vol. XXII, núm. 63, pp. 9-45 <http://www.redalyc.org/articulo. oa?id=13836846001> [ 19 de abril de 2018]

QuiJano, AníBal

2004 "El 'movimiento indígena' y las cuestiones pendientes en América Latina”, en Aníbal Quijano, Cuestiones y horizontes. De la dependencia histórico-estructural a la colonialidad/ descolonialildad del poder, Consejo Latinoamericano de Ciencias Sociales, Buenos Aires, pp. 635-663.

Quintero, MARIETA

y EloÍsa VASCO

2007 "Justificaciones y sentimientos morales de jóvenes universitarios y jóvenes desplazados 
acerca de las acciones justas e injustas", en Acta Colombiana de Psicología, vol. 10, núm. 1, pp. 99-110 <http: / / www.redalyc.org/pdf/ 798/79810110.pdf> [ 19 de abril de 2018].

Reguillo, Rossana

2012 Culturas juveniles, formas políticas del desencanto, Siglo Xxi Editores, Buenos Aires.

Santos, Boaventura de Sousa

2001 "Los nuevos movimientos sociales", en OSAL, septiembre, pp. 177-188.

Santos, Boaventura de Sousa

2006 Conocer desde el Sur. Para una cultura política emancipatoria, Universidad Nacional Mayor de San Marcos, Fondo Editorial de la Facultad de Ciencias Sociales/Programa de Estudios sobre Democracia y Transformación Global, Lima <http: / / www.boaventuradesousasantos.pt/media / Conocer\%20desde\% 20el\%20 Sur_Lima_2006.pdf> [10 febrero de 2018]. Smelser, NeIL

1962 Theory of Collective Behavior, Macmillan, Nueva York.

VAldeZ, ANDRÉs,

DELIA A. Huerta

y Sergio Díaz

2014 "La vergüenza y el orgullo en la política electoral. El caso del sistema de partidos políticos y los medios de comunicación en México", en Revista Enfoques, vol. XII, núm. 20, pp. 115-132. 